\title{
FITORREMEDIAÇÃO DE SOLO CONTAMINADO COM TRIFLOXYSULFURON-SODIUM POR DIFERENTES DENSIDADES POPULACIONAIS DE FEIJÃO-DE-PORCO (Canavalia ensiformis (L). DC.)
}

\author{
Phytoremediation of the herbicide trifloxysulfuron-sodium by different \\ populacional densities of jack bean (Canavalia ensiformis (L). DC.)
}

\author{
José Barbosa dos Santos ${ }^{1}$, Sergio de Oliveira Procópio ${ }^{2}$, Fábio Ribeiro Pires ${ }^{2}$ \\ Antônio Alberto da Silva ${ }^{3}$, Edson Aparecido dos Santos ${ }^{4}$
}

\begin{abstract}
RESUMO
Objetivou-se neste trabalho avaliar a fitorremediação do solo contaminado pelo herbicida trifloxysulfuron-sodium a campo, pela espécie de adubo verde Canavalia ensiformis (feijão-de-porco) cultivada em diferentes densidades populacionais. O delineamento experimental utilizado foi o de blocos casualizados, em esquema fatorial 4 × 2, com quatro repetições. Os tratamentos foram

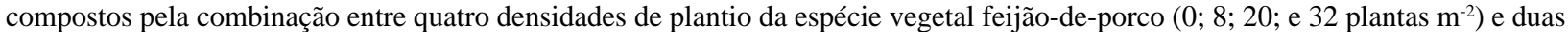
doses do trifloxysulfuron-sodium $\left(0,00 \mathrm{e} 15,00 \mathrm{~g} \mathrm{ha}^{-1}\right)$. Cinco dias após o preparo do solo, o trifloxysulfuron-sodium foi aplicado antes da semeadura da espécie fitorremediadora, a qual foi mantida na área por 65 dias. Após esse período a área experimental foi novamente sulcada e fertilizada sendo cultivado feijão (Phaseolus vulgaris L. cv. Vermelho Coimbra), espécie sensível ao trifloxysulfuron-sodium. Aos 45 dias após a semeadura do feijão avaliou-se a altura e a biomassa seca da parte aérea das plantas e ao final do ciclo da cultura, foram determinados o rendimento de grãos, o número de vagens por planta e o peso de 100 sementes. $\mathrm{O}$ cultivo prévio de $C$. ensiformis nas densidades populacionais de 8,20 ou 32 plantas $\mathrm{m}^{-2}$ promoveu a remediação do herbicida. A densidade populacional mínima de $C$. ensiformis que proporciona maior rendimento de grãos à cultura do feijão é de 20 plantas m ${ }^{-2}$.
\end{abstract}

Termos para indexação: Adubos verdes, biorremediação, descontaminação, Phaseolus vulgaris.

\begin{abstract}
This study aimed to evaluate the phytoremediation of the herbicide trifloxysulfuron-sodium in field, by Canavalia ensiformis cultivated in different populacional density. The experiment was arranged in a randomized block design, in a factorial scheme $4 \times 2$, with four replicates. The treatments consisted of the combination of four sowing densities $\left(0 ; 8 ; 20\right.$; and $\left.32 \mathrm{plants}^{-2}\right)$ and two rates of trifloxysulfuron-sodium ( 0.00 and $\left.15.00 \mathrm{~g} \mathrm{ha}^{-1}\right)$. Five days after soil preparation trifloxysulfuron-sodium was applied, before $C$. ensiformis sowing, which was kept for 65 days in the area. After this time the experimental area was furrowed, fertilized and bean (Phaseolus vulgaris cv. Vermelho Coimbra), specie sensitive to trifloxysulfuron-sodium, was cultivated. After 45 days of bean sowing, plant height and shoot dry biomass were evaluated and at the end of final cycle, grain yield, number of pods per plant and

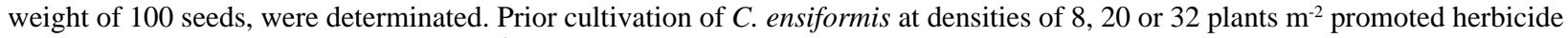
remediation. The minimal of 20 plants $\mathrm{m}^{-2}$ of $C$. ensiformis provides the highest grain yield.
\end{abstract}

Index terms: Biorremediation, decontamination, green manure, Phaseolus vulgaris.

(Recebido para publicação em 1 de janeiro de 2005 e aprovado em 31 de janeiro de 2006)

\section{INTRODUÇÃO}

A presença de herbicidas no solo por longo tempo, além de causar sérios impactos na microbiota do solo (TSUI \& CHU, 2003) e contaminação de água subterrânea, pode inviabilizar a rotação de culturas quando estão envolvidas espécies sensíveis ao princípio ativo (PROCÓPIO et al., 2004; SANTOS et al., 2004a,b). Visando reduzir o período de residualidade de alguns xenobióticos no solo, como herbicidas, tem-se pesquisado o emprego da fitorremediação em sistemas agrícolas. Na tentativa de diminuir o tempo necessário entre a aplicação de herbicidas e o plantio de espécies não-tolerantes a estes, algumas espécies vegetais já foram selecionadas com potencial para a remediação dos herbicidas trifloxysulfuron-sodium (PROCÓPIO et al., 2004; SANTOS et al., 2004a,b) e tebuthiuron (PIRES et al., 2003a,b). Essa antecipação representa importante alternativa de manejo, principalmente em sistemas intensivos de exploração, em que se pratica de dois a três cultivos por ano.

A fitorremediação de herbicidas pode se dar notadamente por dois mecanismos: rizodegradação ou fitoestimulação e fitodegradação ou fitotransformação (SCHNOOR \& DEE, 1997). O primeiro caso ocorre em nível

\footnotetext{
${ }^{1}$ Engenheiro Agrônomo, bolsista pós-doutor do Departamento de Fitotecnia da Universidade Federal de Viçosa/UFV - 36.570-000 - Viçosa, MG jbarbosasantos@yahoo.com.br

Engenheiro Agrônomo, Professor do Departamento de Agronomia da Fundação do Ensino Superior de Rio Verde/FESURV - Cx. P. 104 - $75.901-970$ Rio Verde, GO - soprocopio@yahoo.com.br; frpires@fesurv.br

${ }^{3}$ Engenheiro Agrônomo, Professor do Departamento de Fitotecnia da Universidade Federal de Viçosa/UFV - 36.570-000 - Viçosa, MG.

${ }^{4}$ Discente do Curso de Agronomia da Universidade Federal de Viçosa/UFV - Viçosa, MG - edsonapsant@yahoo.com.br
} 
rizosférico, realizado por microrganismos, associados às raízes de plantas tolerantes, cuja ação é estimulada pela exsudação radicular dessas espécies (CUNNINGHAM et al., 1996). A fitotransformação, por sua vez, se dá pela absorção com subseqüente volatilização, ou degradação parcial ou completa, com transformação em compostos menos tóxicos, combinados e/ou ligados nos tecidos das plantas (ACCIOLY \& SIQUEIRA, 2000). Em geral, a maioria dos compostos orgânicos passa por alguma transformação nas células das plantas antes de ser isolada em vacúolos ou ligar-se a estruturas celulares insolúveis, como a lignina (SALT et al., 1998). Em ambos os mecanismos, a presença de raízes é determinante. Portanto, espécies selecionadas para fitorremediação de herbicidas devem apresentar sistema radicular vigoroso, agressivo e profundo (NEWMAN et al., 1998), e, além disso, elevada taxa transpiratória, responsável pelo fluxo de substâncias das raízes para a parte aérea (SCHNOOR \& DEE, 1997).

Aumentos da densidade populacional de plantas remediadoras em determinada área, até certo limite, também pode proporcionar maior volume de raízes e de solo explorado, podendo resultar em incremento da absorção/ degradação do contaminante e/ou degradação rizosférica. Apesar disso, poucos trabalhos de pesquisa com fitorremediação avaliam a densidade mínima de plantas em que se obtenha o máximo de remediação do solo. Comumente, utiliza-se densidade maior que aquela recomendada para o cultivo comercial das espécies, quando se trata de espécie cultivada (LINDSAY et al., 2003). A densidade adequada dependerá primordialmente da espécie utilizada, porém, de acordo com Schnoor \& Dee
(1997), que trabalharam com espécies arbóreas como agentes fitorremediadores, alta densidade populacional no início de seu estabelecimento assegura taxa significativa de evapotranspiração, o que normalmente é desejável, até certo ponto, a partir do qual a competição intraespecífica pode prejudicar o desenvolvimento das plantas.

O feijão-de-porco, além de apresentar características desejáveis como adubo verde, tem sido testado e aprovado como espécie remediadora de solos contaminados com herbicidas (PIRES et al., 2003a,b), dentre eles, o trifloxysulfuron-sodium (SANTOS et al., 2004b).

Com este trabalho, objetivou-se avaliar a fitorremediação de solo contaminado com o trifloxysulfuron-sodium a campo, exercida por plantas de Canavalia ensiformis, cultivadas em diferentes densidades populacionais.

\section{MATERIAL E MÉTODOS}

O experimento foi realizado na Estação Experimental de Coimbra - MG, em um Argissolo Vermelho-Amarelo, de textura argilo-arenosa, cuja caracterização físico-química encontra-se na Tabela 1 . O período de condução do experimento a campo foi de março a novembro de 2003.

$\mathrm{O}$ delineamento experimental utilizado foi o de blocos casualizados em esquema fatorial 4 x 2 , com quatro repetições. Os tratamentos foram compostos pela combinação entre quatro densidades de plantio da espécie vegetal feijão-deporco $\left(0 ; 8 ; 20\right.$; e 32 plantas $\mathrm{m}^{-2}$, o que corresponde a zero; $1 \mathrm{x}$; 2,5x; e 4x a densidade recomendada na adubação verde) e duas doses do herbicida trifloxysulfuron-sodium [0,00 e 15,00 $\mathrm{g} \mathrm{ha}^{-1}$ (equivalente a duas vezes a dose recomendada para uso na cultura do algodão)].

TABELA 1 - Composição físico-química da camada arável $(0-20 \mathrm{~cm})$ do solo Argissolo Vermelho-Amarelo utilizado no experimento. Coimbra, MG. 2003.

\begin{tabular}{|c|c|c|c|c|c|c|c|c|c|c|}
\hline \multicolumn{11}{|c|}{ Análise granulométrica $\left(\mathrm{dag} \mathrm{kg}^{-1}\right)$} \\
\hline \multicolumn{3}{|c|}{ Argila } & \multicolumn{2}{|c|}{ Silte } & Areia fina & \multicolumn{2}{|c|}{ Areia grossa } & \multicolumn{3}{|c|}{ Classificação textural } \\
\hline \multicolumn{3}{|c|}{34} & \multicolumn{2}{|c|}{12} & \multicolumn{2}{|l|}{18} & 36 & \multicolumn{3}{|c|}{ Franco-Argilo-arenoso } \\
\hline \multicolumn{11}{|c|}{ Análise Química } \\
\hline $\mathrm{pH}$ & $\mathrm{P}$ & $\mathrm{K}^{+}$ & $\mathrm{H}+\mathrm{Al}$ & $\mathrm{Al}^{3+}$ & $\mathrm{Ca}^{2+}$ & $\mathrm{Mg}^{2+}$ & $\begin{array}{c}\text { CTC } \\
\text { total }\end{array}$ & $\mathrm{V}$ & M & MO \\
\hline $\mathrm{H}_{2} \mathrm{O}$ & \multicolumn{2}{|c|}{$\mathrm{mg} \mathrm{dm}^{-3}$} & \multicolumn{5}{|c|}{$\mathrm{Cmol}_{\mathrm{c}} \mathrm{dm}^{-3}$} & \multicolumn{2}{|c|}{$\%$} & dag $\mathrm{kg}^{-1}$ \\
\hline 5,5 & 7,4 & 76 & 2,7 & 0,0 & 2,8 & 0,8 & 3,61 & 56 & 0 & 3,68 \\
\hline
\end{tabular}

*Análises realizadas nos Laboratórios de Análises Físicas e Químicas de Solo do Departamento de Solos da UFV, segundo a metodologia descrita pela Empresa Brasileira de Pesquisa Agropecuária (EMBRAPA, 1997). 
Antes da semeadura foi realizado o preparo convencional do solo, constituindo-se de uma aração e duas gradagens. Após isso, o trifloxysulfuron-sodium foi aplicado nas devidas parcelas, utilizando-se um pulverizador costal pressurizado com gás carbônico $\left(\mathrm{CO}_{2}\right)$, aplicando o equivalente a $200 \mathrm{~L} \mathrm{ha}^{-1}$ de calda.

O espaçamento utilizado para a semeadura do feijãode-porco foi de $0,30 \mathrm{~m}$, sendo essa operação realizada em sulcos, manualmente. Após, aproximadamente, 20 dias da semeadura, foi realizado um desbaste deixando-se na área o número de plantas pré-estabelecidas, de acordo com o tratamento até aos 65 dias, quando foram cortadas rente ao solo, sendo sua parte aérea deixada sobre a superfície da parcela experimental, durante 15 dias. Ao final desse período, a área experimental foi novamente sulcada e fertilizada, sendo cultivado o feijão ( $P$. vulgaris cv. Vermelho Coimbra), espécie sensível ao herbicida avaliado no espaçamento de $0,50 \mathrm{~m}$, sendo semeadas manualmente 10 sementes por metro. Durante todo o experimento (ciclo do feijão-de-porco mais ciclo do feijão) a área de todas as parcelas foi mantida livre de plantas daninhas, por meio de capinas manuais.

Aos 45 dias após a semeadura (DAS) das plantas de feijão avaliou-se a altura de plantas, adotando-se como base para medição o meristema apical, e a biomassa seca da parte aérea de cinco plantas colhidas ao acaso por parcela, obtida por meio de pesagem do material colhido, secado em estufa de circulação forçada $\left(70 \pm 2{ }^{\circ} \mathrm{C}\right)$ por 72 horas. Também, ao final do ciclo da cultura, foram determinados o rendimento de grãos, o número de vagens por planta e o peso de 100 sementes.

Todas as variáveis que atenderam às pressuposições de normalidade e homogeneidade das variâncias, por meio dos testes de Lilliefors e de Cochran, respectivamente, foram submetidas à análise de variância, sendo o efeito entre as densidades de plantas de feijãode-porco avaliado por análise de regressão, a 1 ou $5 \%$ de significância.

\section{RESULTADOS E DISCUSSÃO}

Quando não se cultivou feijão-de-porco (densidade zero de plantas) anteriormente à semeadura do feijão observou-se redução na altura de plantas cultivadas em solo com a aplicação prévia do trifloxysulfuron-sodium (Tabela 2). Todavia, com o cultivo prévio desse adubo verde, independente da densidade populacional adotada, verifica-se que não houve diminuição na altura de plantas de feijão cultivadas em solo pré-contaminado com o herbicida. Observou-se, inclusive, ocorrência de plantas com maior altura se desenvolvendo nestas condições quando cultivadas em parcelas que anteriormente continham oito plantas $\mathrm{m}^{-2} \mathrm{de}$ feijão-de-porco (Tabela 2). Esse resultado corrobora o verificado por Santos et al. (2004b), que não observaram a redução da altura de plantas de milho cultivadas em solo contaminado por vários níveis de trifloxysulfuronsodium, quando anteriormente se cultivou feijão-deporco. Todavia, constatou-se que o cultivo de feijão-deporco na densidade de 32 plantas $\mathrm{m}^{-2}$, tanto no solo que recebeu previamente o herbicida, como também onde não houve aplicação, provocou redução na altura de plantas de feijão (Figura 1).

Quanto ao acúmulo de biomassa pelas plantas de feijão, o cultivo prévio de feijão-de-porco não evitou o efeito negativo do trifloxysulfuron-sodium no solo (Tabela 2). Resultados semelhantes foram observados por Santos et al. (2004b), os quais constataram redução da biomassa seca de plantas milho cultivadas em solo que recebeu aplicação de $15 \mathrm{~g} \mathrm{ha}^{-1}$ de trifloxysulfuron-sodium, cultivadas após a semeadura de feijão-de-porco. Todavia, essa redução foi menos acentuada em relação ao observado quando não se realizou o cultivo prévio desse adubo verde. $\mathrm{O}$ aumento da densidade populacional das plantas de feijão-de-porco, cultivadas nas parcelas onde não foi realizada aplicação prévia do herbicida, provocou redução da biomassa seca da parte aérea das plantas de feijão (Figura 1), fato este, também observado nas parcelas onde se aplicou o produto para as densidades superiores a oito plantas de feijão-de-porco por metro quadrado.

A aplicação prévia no solo de trifloxysulfuronsodium, ou o cultivo anterior na área de diferentes densidades populacionais de feijão-de-porco, não afetaram no peso de 100 sementes (Tabela 2).

O cultivo de feijão-de-porco na área contaminada com o herbicida antes da semeadura de feijão, nas densidades populacionais de 20 e 32 plantas por metro quadrado, evitou que o número de vagens por planta fosse reduzido (Tabela 2). Foi observado para o tratamento que manteve 20 plantas $\mathrm{m}^{-2}$ do adubo verde, cultivado no solo tratado com trifloxysulfuron-sodium, maior número de vagens planta $^{-1}$ de feijão (Figura 1). $\mathrm{Na}$ área sem contaminação prévia pelo herbicida não se verificou efeito da densidade populacional de plantas de feijão-de-porco para esse parâmetro.

Ciênc. agrotec., Lavras, v. 30, n. 3, p. 444-449, maio./jun., 2006 
TABELA 2 - Altura de plantas e biomassa seca da parte aérea aos 45 dias após a semeadura, peso de 100 sementes, número de vagens por planta e rendimento de grãos de feijão (cultivar Vermelho-Coimbra) semeado após o cultivo prévio de Canavalia ensiformis, em diferentes densidades populacionais, em solo contaminado com o herbicida trifloxysulfuron-sodium (15 $\left.\mathrm{g} \mathrm{ha}^{-1}\right)$. Coimbra, MG. 2003

\begin{tabular}{|c|c|c|c|c|}
\hline \multirow{2}{*}{$\begin{array}{c}\text { Densidade } \\
\left(\text { plantas } \mathbf{m}^{-2}\right)\end{array}$} & \multicolumn{2}{|c|}{ Altura de plantas (cm) } & \multicolumn{2}{|c|}{ Biomassa seca da parte aérea $(\mathrm{g})$} \\
\hline & Solo com herbicida & Solo sem herbicida & Solo com herbicida & Solo sem herbicida \\
\hline 0 & $87,40 \mathrm{~b}$ & $93,32 \mathrm{a}$ & $21,25 \mathrm{~b}$ & $28,85 \mathrm{a}$ \\
\hline 8 & 98,00 a & $94,17 \mathrm{~b}$ & $23,65 \mathrm{~b}$ & $28,02 \mathrm{a}$ \\
\hline 20 & 96,70 a & $98,00 \mathrm{a}$ & $21,50 \mathrm{~b}$ & $26,97 \mathrm{a}$ \\
\hline 32 & $88,17 \mathrm{a}$ & $88,90 \mathrm{a}$ & $19,31 \mathrm{~b}$ & $22,72 \mathrm{a}$ \\
\hline \multirow[t]{3}{*}{ C.V. $(\%)$} & ---------------------3, & 47-------------------- & $\begin{array}{l}-------------------5 \\
\end{array}$ & 49-------------------- \\
\hline & \multicolumn{2}{|c|}{ Peso 100 sementes (g) } & \multicolumn{2}{|c|}{ Vagens planta $^{-1}$} \\
\hline & Solo com herbicida & Solo sem herbicida & Solo com herbicida & Solo sem herbicida \\
\hline 0 & $22,46 \mathrm{a}$ & $21,64 \mathrm{a}$ & $6,18 \mathrm{~b}$ & $10,10 \mathrm{a}$ \\
\hline 8 & 20,99 a & 20,96 a & $9,54 \mathrm{~b}$ & $11,50 \mathrm{a}$ \\
\hline 20 & $22,82 \mathrm{a}$ & 22,77 a & $11,14 \mathrm{a}$ & $10,85 \mathrm{a}$ \\
\hline 32 & $22,63 \mathrm{a}$ & $22,35 \mathrm{a}$ & $9,56 \mathrm{a}$ & $10,35 \mathrm{a}$ \\
\hline \multirow[t]{3}{*}{ C.V. $(\%)$} & --------------------4, & 21-------------------- & 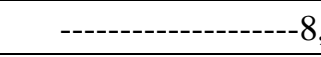 & 99--------------------- \\
\hline & \multicolumn{4}{|c|}{ Rendimento de grãos $\left(\mathrm{kg} \mathrm{ha}^{-1}\right)$} \\
\hline & \multicolumn{2}{|c|}{ Solo com herbicida } & \multicolumn{2}{|c|}{ Solo sem herbicida } \\
\hline 0 & \multicolumn{2}{|c|}{$2285,92 \mathrm{~b}$} & \multicolumn{2}{|c|}{$2408,85 \mathrm{a}$} \\
\hline 8 & \multicolumn{2}{|c|}{$2437,11 \mathrm{a}$} & \multicolumn{2}{|c|}{$2415,36 \mathrm{a}$} \\
\hline 20 & \multicolumn{2}{|c|}{$2548,97 \mathrm{a}$} & \multicolumn{2}{|c|}{$2455,73 \mathrm{a}$} \\
\hline 32 & \multicolumn{2}{|c|}{$2518,42 \mathrm{a}$} & \multicolumn{2}{|c|}{$2506,38 \mathrm{a}$} \\
\hline
\end{tabular}

Médias seguidas por letras iguais, na linha, para cada característica, não diferem entre si pelo F a 5\% de probabilidade.

O cultivo prévio de feijão-de-porco nas densidades populacionais de 8,20 ou 32 plantas $\mathrm{m}^{-2}$ impediu a perda de rendimento de grãos de feijão decorrentes do residual do herbicida trifloxysulfuron-sodium no solo (Tabela 2), visto que tal perda apenas ocorreu nas parcelas onde não se realizou o cultivo anterior do adubo verde (densidade zero). Wilson et al. (2000) constataram que a atividade do herbicida simazine em solução foi reduzida a 45 e $34 \%$, em sete dias, com a presença de Acorus gramenius L. e Pontederia cordata L., respectivamente. Nas parcelas onde não houve a aplicação do herbicida, observou-se que o incremento no número de plantas de feijão-de-porco cultivadas na área proporcionou aumento do rendimento de grãos de feijão (Figura 1). Tal fato, também ocorreu nas áreas onde foi aplicado o trifloxysulfuron-sodium, porém até a densidade de 20 plantas $\mathrm{m}^{-2}$ de feijão-de-porco. 

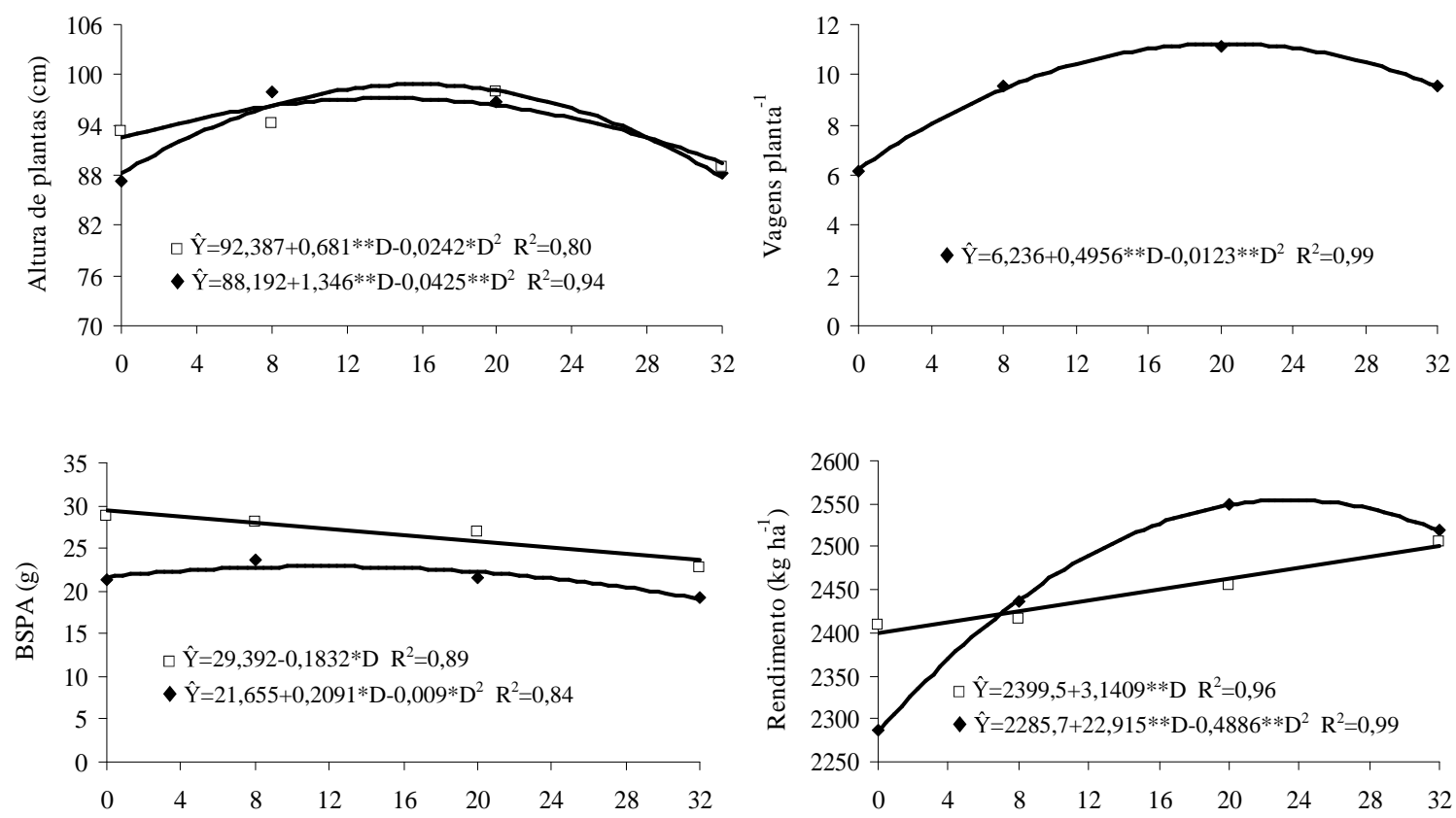

Densidade populacional de Canavalia ensiformis (plantas $\mathrm{m}^{-2}$ )

FIGURA 1 - Efeito de diferentes densidades populacionais de Canavalia ensiformis, utilizadas para remediação de solo contaminado com o herbicida trifloxysulfuron-sodium $\left(15 \mathrm{~g} \mathrm{ha}^{-1}\right)$ sobre a altura de plantas e a biomassa seca da parte aérea (BSPA), aos 45 dias após a semeadura, número de vagens por planta e rendimento de grãos de feijão (cultivar Vermelho-Coimbra). ( $\downarrow$ ) com herbicida,( $\square)$ sem herbicida. * e **: significativo a 5\% e $1 \%$ de probabilidade de erro, respectivamente, pelo teste $t$.

\section{CONCLUSÕES}

O feijão-de-porco é eficiente na remediação do solo contaminado com o herbicida trifloxysulfuron-sodium, em condições de campo. A densidade populacional mínima desse adubo verde, que possibilita o desenvolvimento e produtividade do feijoeiro, deve ser de 20 plantas $\mathrm{m}^{-2}$.

\section{AGRADECIMENTOS}

À Empresa Syngenta Proteção de Cultivos Ltda., pelo apoio à execução do projeto.

\section{REFERÊNCIAS BIBLIOGRÁFICAS}

ACCIOLY, A. M. A.; SIQUEIRA, J. O. Contaminação química e biorremediação do solo. In: NOVAIS, R. F. et al. (Eds.). Tópicos em ciência do solo. Viçosa: Sociedade Brasileira de Ciência do Solo, 2000. v. 1, p. 299-352.
CUNNINGHAM, S. D. et al. Phytoremediation of soils contaminated with organic pollutants. Advances in Agronomy, San Diego, v. 56, p. 55-114, 1996.

EMPRESA BRASILEIRA DE PESQUISA AGROPECUÁRIA. Centro Nacional de Pesquisa de Solos. Manual de métodos de análise de solo. 2. ed. Rio de Janeiro, 1997. $212 \mathrm{p}$.

LINDSAY, E. B. et al. Analysis of transgenic indian mustard plants for phytoremediation of metal-contaminated mine tailings. Journal of Environmental Quality, [S.1.], v. 32, p. 432-440, 2003.

NEWMAN, L. A. et al. Phytoremediation of organic contaminants: a review of phytoremediation research at the University of Washington. Journal of Soil Contaminant, [S.1.], v. 7, p. 531-542, 1998. 
PIRES, F. R. et al. Seleção de plantas tolerantes ao tebuthiuron e com potencial para fitorremediação. Revista Ceres, Viçosa, v. 20, p. 583-594, 2003 a.

PIRES, F. R. et al. Seleção de plantas com potencial para fitorremediação de tebuthiuron. Planta Daninha, Rio de Janeiro, v. 21, p. 451-458, 2003 b.

PROCÓPIO, S. O. et al. Seleção de plantas com potencial para fitorremediação de solos contaminados com o herbicida trifloxysulfuron sodium. Planta Daninha, Rio de Janeiro, v. 22, p. 315-322, 2004.

SALT, D. E. et al. Phytoremediation. Annual Review Plant Physiology, Pato Alto, v. 49, p. 643-668, 1998.

SANTOS, J. B. et al. Seletividade do herbicida trifloxysulfuron-sodium para fins de fitorremediação. Revista Ceres, Viçosa, v. 51, p. 129-141, 2004a.
SANTOS, J. B. et al. Fitorremediação do herbicida trifloxysulfuron sodium. Planta Daninha, Rio de Janeiro, v. 22, p. 323-330, 2004 b.

SCHNOOR, J. L.; DEE, P. E. Technology evaluation report: phytoremediation. Pittsburgh: Ground-Water Remediation Technologies Analysis Center, 1997. 37 p. (E Series: GWRTAC TE-98-01).

TSUI, M. T. K.; CHU, L. M. Aquatic toxicity of glyphosatebased formulations: comparison between different organisms and the effects of environmental factors.

Chemosphere, [S.1.], v. 52, p. 1189-1197, 2003.

WILSON, P. C.; WHITWELl, T.; KLAINE, S. J. Phytotoxicity, uptake, and distribution of ${ }^{14} \mathrm{C}$-simazine in Acorus gramenius and Pontederia cordata. Weed Science, Champaign, v. 48, p. 701-709, 2000. 